\title{
La equidad como elemento fundamental de los procesos de delimitación marítima en la jurisprudencia de la Corte Internacional de Justicia**
}

\section{Equity as the fundamental element in maritime delimitation cases in the International Court of Justice Jurisprudence}

SUMARIO

Introducción. 1. La equidad y el derecho natural. 2. La equidad como principio universal. 3. La equidad en el derecho internacional de delimitación marítima. 4. La operación adjudicativa de delimitación con equidad. 4.1. Línea media provisional. 4.2. Circunstancias pertinentes 4.3. El análisis de desproporcionalidad. Conclusiones. Referencias.

RESUMEN

La Corte Internacional de Justicia, con ocasión del ejercicio de su función contenciosa, ha tenido que abordar numerosas controversias internacionales sobre delimitaciones territoriales y marítimas en prácticamente todos los continentes del mundo. Lo anterior le ha permitido desarrollar, en el ámbito de derecho internacional del mar, normas consuetudinarias de delimitación basadas en la equidad infra legem, que tiene como objetivo y fin la equidad de resultados.

PALABRAS CLAVE

Corte Internacional de Justicia, equidad, delimitación marítima.

*Abogado de la Universidad Externado de Colombia. Magíster en Derecho Internacional de la Universidad Católica de Lovaina y con estudios de maestría en Cooperación Internacional y Relaciones Exteriores. Docente investigador en Derecho Internacional Público de la Universidad Externado de Colombia (Bogotá, Colombia).Contacto: wilfredo.robayo@uexternado.edu.co

** Recibido el 21 de septiembre de 2016, aprobado el 1. ${ }^{\circ}$ de marzo de 2017.

Para citar el artículo: Robayo GaLvis, W. La equidad como elemento fundamental de los procesos de delimitación marítima en la jurisprudencia de la Corte Internacional de Justicia. Derecho del Estado n. ${ }^{\circ} 38$, Universidad Externado de Colombia, enero-junio de 2017, pp. 199221. DoI: https://doi.org/10.18601/01229893.n38.08 
ABSTRACT

The International Court of Justice in exercising its contentious jurisdiction has had to deal with several international disputes concerning territorial and maritime delimitations in every continent in the world. This has enabled the Court, under the International Law of the Sea scope, to identify and develop some customary rules of delimitation based in equity infra legem, which aims to attain equitable results.

\section{KEYWORDS}

International Court of Justice, equity, maritime delimitation.

\section{INTRODUCCIÓN}

A lo largo de la historia algunos Estados han mostrado que uno de sus mayores intereses se centra en la adquisición y conservación del territorio, y es a partir de esta premisa que podemos entender que con el pasar del tiempo la jurisdicción estatal poco a poco se ha expandido a nuevos espacios que en principio se encontraban fuera del alcance de la soberanía estatal. Pues bien, una situación que ejemplifica la afirmación precedente es la relacionada con los dominios marinos.

La historia del derecho internacional del mar refleja fuertes discusiones doctrinales iniciadas en el siglo XVII, que manifiestan la preocupación que desde la Antiguiedad hasta tiempos presentes ha existido con respecto al dominio del mar. Prueba de lo anterior la encontramos en la propuesta de Francisco de Vitoria, quien proponía la teoría de la inapropiabilidad de los espacios marinos, tesis que sería reforzada posteriormente por Hugo Grocio ${ }^{1}$, quien pregonaba la libertad de los mares, bajo el entendido de la imposibilidad de ocupar y delimitar una situación o cosa especialmente ilimitada como los océanos. Por su parte, John Selden, en su obra Mare clausum, defiende contrariamente la posibilidad de apropiación de los mares, permitiéndoles a los Estados ejercer dominio sobre los espacios marinos ${ }^{2}$.

Bajo ese entendido, intereses de tipo económico, político y comercial permitieron a los Estados extender su soberanía en los mares, situación que a todas luces generaría controversias internacionales que deberían ser resueltas bien a través de negociaciones entre los Estados, delimitando sus espacios marítimos y, a su vez, estableciendo derechos y obligaciones en

1 Grocio, H. De la libertad de los mares. Trad. V. Blanco García y L. García Arias. Madrid: Centro de Estudios Constitucionales, 1979.

2 VÁsquez CARrizosa, A. El nuevo derecho del mar. Evolución y proyecciones económicas. Bogotá: Temis, 1986, 3-28; B ARDONnET, D., dir. Le nouveau droit de la mer. Paris: Pedone, 1983; Dupuy, R.J. Traité du nouveau droit de la mer. Paris: Economica-Bruylant, 1985. 
tratados internacionales, o bien mediante la aplicación de las disposiciones del derecho internacional a través de alguno de los mecanismos de solución pacífica de controversias ${ }^{3}$.

Asimismo es importante tener en cuenta que a medida que los años pasan, los Estados, y en general la humanidad, presentan avances tecnológicos y científicos que les permite explorar cada vez más el planeta y al mismo tiempo encontrar nuevas formas de riqueza, situación que justifica aún más los intereses de los Estados por la apropiación y goce de los espacios marinos, donde la existencia de recursos naturales renovables y no renovables es el mayor atractivo y motivación para el desarrollo de procesos de delimitación marítima entre los Estados.

En consecuencia, poco a poco se fueron creando normas internacionales convencionales ${ }^{4} \mathrm{y}$ consuetudinarias, cuyos fines buscan establecer las áreas marítimas sobre las cuales los Estados pueden ejercer soberanía, así como establecer la extensión de las mismas. De esta manera se van constituyendo espacios como el mar territorial, la zona contigua, la zona económica exclusiva y la plataforma continental. Si bien estos conceptos son aceptados por algunos miembros de la comunidad internacional, dicha aceptación parcial supuso la transformación de estas áreas, sobre todo en lo que se refiere a la extensión de las mismas.

Por todo lo anterior, la evolución experimentada por el derecho internacional del mar y por ende por las nuevas zonas marinas implicó que las relaciones entre los Estados encontraran un nuevo foco de tensión, con respecto a las extensiones y/o anchuras de aquellas, pero sobre todo frente a la superposición espacial de sus derechos. Bajo este escenario las operaciones de delimitación de los espacios marítimos aumentan su importancia en el escenario internacional, en la medida que son fuente de controversias internacionales ${ }^{5}$. Así pues, los procesos de delimitación buscan establecer líneas divisorias en el mar, que permitan distinguir las áreas donde los Estados ejercen sus poderes

3 Por supuesto que la afirmación esbozada debe ser analizada bajo el entendido de que a lo largo de la historia del derecho internacional los Estados también gozaban de la libertad de hacer la guerra, la cual era ampliamente utilizada para la solución de las controversias internacionales. Sin embargo, es importante tener en cuenta que los Estados usaban de manera frecuente otros mecanismos diferentes al uso de la fuerza para dar por terminados los conflictos que se suscitaban en sus relaciones internacionales, tales como la negociación directa, la mediación, la conciliación e incluso mecanismos de índole jurisdiccional.

4 Dentro de ellas podemos destacar las cuatro Convenciones de Ginebra sobre el Derecho del Mar de 1958, discriminadas de la siguiente manera: 1. La Convención sobre Mar Territorial y Zona Contigua, que entró en vigor el 10 de septiembre de 1964; 2. La Convención sobre Alta Mar, que entró en vigor el 30 de septiembre de 1962; 3. La Convención sobre Plataforma Continental, que entró en vigor el 10 de junio de 1964, y 4. La Convención sobre Pesca y Conservación de los Recursos Vivos de la Alta Mar, que entró en vigor el 20 de marzo de 1966. Asimismo se destaca la Convención de las Naciones Unidas sobre el Derecho del Mar de 1982 (CONVEMAR).

5 Anderson, D. Modern Law of the Sea: Selected Essays. Leiden: Martinus Nijhoff, 2008,382 . 
soberanos, determinando catálogos de derechos y obligaciones, lo que se traduce en la asignación de competencias al Estado territorial y la exclusión de pretensiones de terceros Estados ${ }^{6}$.

Así las cosas, la delimitación de las zonas marítimas adquiere gran relevancia en el escenario internacional, obligando tanto a los Estados como a los tribunales internacionales y a los estudiosos del derecho internacional a examinar con detalle la situación. De esta manera salta a la vista la cuestión que puede resultar como la mayor preocupación, y es aquella que se refiere al método de delimitación que se elegirá al momento de abordar un proceso de determinación de límites marítimos entre Estados. En ese orden de ideas, tratándose de negociaciones entre Estados, el inconveniente será resuelto por ellos mismos, es decir, en el tratado internacional de delimitación marítima; serán los Estados parte quienes determinen el método a utilizar. Sin embargo, no ocurre lo mismo en el contencioso internacional, pues si bien los Estados en sus pretensiones proponen a los tribunales la manera como debe llevarse a cabo el procedimiento, el juez internacional cumple su labor atendiendo a lo que dicta el derecho internacional aplicable, ya sea convencional o consuetudinario.

En ese orden de ideas es posible afirmar que el mayor reto que se presenta al respecto es la poca claridad de las normas internacionales convencionales que sobre la materia se han establecido, quedando el juez internacional obligado a echar mano de la costumbre internacional, que pregona el uso de la equidad en los procesos de delimitación marítima. Así pues, debemos cuestionarnos sobre cuáles son los alcances de la equidad en esta labor, y cuál es su papel de cara al derecho internacional.

Pues bien, la problemática planteada en las líneas que preceden ha sido una constante en procesos de delimitación marítima entre Estados a lo largo y ancho de todo el planeta, no pudiendo escapar a esta los Estados de América Latina, quienes en los últimos años han sido testigos de tres fallos de la Corte Internacional de Justicia en los cuales salta a la vista que el protagonista para unos y el antagonista para otros es la equidad.

En razón a lo anterior, el presente escrito tiene como objetivo analizar, a la luz de la jurisprudencia de la Corte Internacional de Justicia, cómo la equidad ha sido utilizada como principio y fin en los procedimientos de delimitación marítima.

\section{LA EQUIDAD Y EL DERECHO NATURAL}

A lo largo de la historia han sido numerosos los filósofos y pensadores que se han cuestionado sobre la relación existente entre justicia y derecho, sur-

6 FRANCK, T. Fairness in International Law Institutions. Oxford: Oxford University Press, $1995,56$. 
giendo así diferentes interrogantes, como por ejemplo: ¿el derecho siempre es justo?, ¿se puede ir más allá de lo establecido en el derecho para llegar a una solución justa? Es posible que la respuestas a estos interrogantes se encuentren en los pensamientos de Aristóteles o incluso en la Biblia, sin embargo, es difícil encontrar una respuesta absoluta.

El término "equidad" viene del latín equitas que significa "igualdad de ánimo". Igualmente podemos encontrar la concepción aristotélica en el término epieikeia. Este último se refiere a la corrección de la ley escrita. Siendo aún más preciso, para Aristóteles las leyes humanas no son omnicomprensivas, es decir, resultan limitadas en la medida que no pueden de manera precedente tener en cuenta todas y cada una de las situaciones que regulan. En esa medida, los hombres podrán corregir los defectos que se presenten en el momento preciso de la aplicación estricta de la ley, y así podrán adaptarla y aplicarla a las situaciones particulares ${ }^{7}$. Así las cosas, es perfectamente posible hacer la distinción entre derecho y equidad, diferenciación que nos permite llegar a una idea de justicia. Bajo esta perspectiva, la equidad no puede considerarse como una regla del derecho, lo que no implica que estos dos conceptos puedan estar separados si queremos acercarnos a una situación justa.

Asimismo podemos encontrar una idea similar en el pensamiento judeocristiano. En ella se incluye un sentimiento de humanidad, de clemencia e incluso de misericordia, que permite crear una relación entre razón y sentimiento ${ }^{8}$. Sin mayores dudas, esta concepción influenció a Hugo Grocio, quien terminó por usar dichos elementos en función de la hermenéutica jurídica.

Sin ir más lejos, podemos observar y concluir que estas visiones mencionadas hacen de la equidad un principio de derecho natural, que apunta no solamente a un orden de la naturaleza, sino a la naturaleza misma del ser humano, propuesta que se opone a la idea de un derecho positivo creado por la sociedad.

Finalmente, vale la pena resaltar desde un enfoque más voluntarista la propuesta de John Rawls, quien propone que una vez los hombres deciden vivir en comunidad, establecen unos límites a su libertad, recibiendo como contrapartida ciertas ventajas derivadas de la obediencia misma del sistema, una contrapartida equitativa. Esto es lo que el autor ha llamado el principio de equidad ${ }^{9}$.

7 Aristóteles. Ética a Nicómaco. Madrid, Tecnos, 2011, Libro v, Cap. X.

8 Falcón y Tella, M. J. Equidad, derecho y justicia. Madrid: Centro de estudios Ramón Aréces, 2005, 203-204.

9 Rawls, J. Teoría de la justicia. México, FCE, 1997, 110-115. 


\section{LA EQUIDAD COMO PRINCIPIO UNIVERSAL}

La noción de equidad y el fundamento mismo que inspira este concepto se encuentran presentes en diversos sistemas jurídicos alrededor de todo el mundo. Es así como la Corte Internacional de Justicia lo ha puesto de presente en el fallo de la Plataforma Continental (Túnez y la Jamahiriya Árabe Libia) al afirmar que "dans l'histoire des systèmes juridiques, le terme équité a servi à désigner diverses notions juridiques. On a souvent opposé l'équité aux règles rigides du droit positif, dont la rigueur doit être temperée pour que justice soit rendue" ${ }^{\prime 10}$. De esta manera podemos destacar la existencia de la equidad en el sistema anglosajón del common law (equity), en el sistema romano-germánico, en el antiguo derecho romano (jus honorarium), como también en sistemas un poco más lejanos como el musulmán, el chino, el hindú, entre otros ${ }^{11}$. No obstante, como bien lo afirma el juez Weeramantry, la proliferación de la equidad en los diferentes sistemas jurídicos del mundo no significa de manera automática e inmediata una adaptación idéntica de la equidad en el derecho internacional ${ }^{12}$.

Así las cosas, tendríamos que preguntarnos: ¿cómo podemos explicar la existencia de la equidad en el derecho internacional? En primer lugar, partiendo de un análisis contemporáneo del sistema jurídico internacional, se debe remarcar que el artículo $1 .^{\circ}$ de la Carta de las Naciones Unidas invita tanto a los Estados de manera individual como a la comunidad internacional en su conjunto a "mantener la paz y la seguridad internacional $[\ldots]$ de conformidad con los principios de la justicia y del derecho internacional". Es ahí donde se encuentra la clave de ingreso de la equidad, estando esta por fuera del derecho internacional, pero incorporándose a aquel, con el fin de llegar a una solución justa.

De igual forma, el Estatuto de la Corte Internacional de Justicia hace mención expresa a la equidad, en su artículo 38, cuando establece que la Corte tiene la facultad de fallar ex aequo et bono, cuando así las partes lo hayan establecido. En consecuencia, esta figura adquiere un carácter excepcional, en la medida que solamente podrá ser utilizada por la disposición de las partes en litigio, y además supone alejarse completamente de las reglas del derecho. Sin embargo, esta opción no parece convencer a los Estados, quienes se muestran aislados de esta posibilidad, ya que, como lo afirma Olivier Pirote, la Corte debe pronunciar el derecho y no construir fallos bajo la idea de una

10 International Court of Justice. Continental shelf (Tunisia/Libya), Judgement, ICJ Reports 1982,60 , pr. 71

11 Ibíd.

12 Cour International de Justice. Délimitation maritime dans la región située entre le Groenland et Jan Mayen (Danemark/Norvège), Arrêt, 14 juin 1993, Recueil CIJ 1993, opinión individuelle M. Weeramantry, § 51, 226. 
suerte de justicia subjetiva, arraigada en reglas morales de contenido vago y fácilmente cuestionables, escenario propicio para el uso de la equidad ${ }^{13}$.

No obstante, a partir de 1969 la jurisprudencia de la Corte Internacional de Justicia incorpora paulatinamente en materia de delimitación marítima la equidad como principio y fin de los procedimientos, empezando allí una importante carrera del concepto en el ordenamiento jurídico internacional.

\section{LA EQUIDAD EN EL DERECHO INTERNACIONAL \\ DE DELIMITACIÓN MARÍTIMA}

Antes de entrar en detalle sobre el aspecto central de este aparte, es importante cuestionarse sobre las características del derecho internacional de delimitación marítima ${ }^{14}$, sobre todo en lo relacionado con su precisión, certeza y capacidad de regulación, frente a los diferentes inconvenientes que pueden presentarse en las relaciones internacionales de los Estados en esta materia. Lo anterior nos lleva a afirmar, como lo ha hecho gran parte de la doctrina, que se trata de una rama del derecho internacional con poco éxito ${ }^{15}$. Los Estados, si bien han unido esfuerzos para regular el tema, lo han hecho con muy pocos los resultados sobresalientes.

Partiendo de la situación anterior, encontramos cierta incertidumbre frente a las controversias entre Estados, cuyo núcleo se centra en la delimitación de los espacios marinos. En primer lugar, se debe a que las normas internacionales establecidas para estos efectos, al igual que muchas otras normas jurídicas en diferentes materias, no tienen la facultad de regular todas las situaciones posibles, sobre todo cuando se trata de territorios de Estados, que a todas luces obedecen a particularidades concretas, derivadas de la configuración geológica y geomorfológica de los espacios susceptibles de ser sometidos a la soberanía estatal. En segundo lugar, obedece a que los tratados internacionales multilaterales que establecen regímenes generales sobre las extensiones de los espacios marinos, así como los derechos y obligaciones de los Estados territoriales, no han sido ratificados por todos los miembros de la comunidad internacional, impidiendo así que les sean vinculantes los preceptos allí establecidos, situación que se refleja con las Convenciones de Ginebra de 1958 y la CONVEMAR de 1982. Y finalmente, este corpus iuris

13 Pirote, O. La notion d'équité dans la jurisprudence récente de la cour international de justice. RGDIP. Paris, t. 77, 1973/1, 93.

14 Atendiendo a los aportes de la doctrina entendemos por delimitación marítima "el proceso de partición equitativa del solapamiento entre las áreas marítimas de dos estados que se encuentran frente a frente o que tienen límites laterales": Rodríguez Cuadros, M. Delimitación marítima con equidad: el caso de Perú y Chile. Lima, Peisa, 2007, 55-58.

15 OrRego VicuÑa, F. El papel de la Corte Internacional de Justicia y otros tribunales en el desarrollo de la delimitación marítima. Estudios Internacionales. Vol. 24, n. ${ }^{\circ} 95$, julio-septiembre, 1991, 384-407. 
internacional que sobre la materia se ha ido poco a poco edificando no ha sido reconocido como derecho consuetudinario en su totalidad, razón por la cual las obligaciones internacionales que allí surgen no poseen vocación de aplicación general.

Pues bien, de cara a un escenario como el recién mencionado es que aparece la equidad en el ámbito del derecho internacional de la delimitación marítima, concepto que debe ser precisado en cuanto a su relación con el ordenamiento jurídico supranacional. Para estos efectos es posible encontrar en la doctrina diferentes clasificaciones en torno al concepto de equidad, guardando especial interés para el objetivo de este trabajo, aquella que distingue entre: equidad contra legem, esto es, aquella que está en plena contradicción con las disposiciones jurídicas; equidad praeter legem, refiriéndose a la que se usa para llenar las lagunas del derecho, es decir, se aplica en defecto de la ley; y por último, equidad infra legem, para hacer mención a aquella que se utiliza dentro del marco mismo del derecho, es decir, la que la norma jurídica contiene.

Es entonces esta última la que guarda estrecha relación con el derecho internacional. La equidad infra legem, también llamada secundum o propter legem, es aquella que tiene un contenido interpretativo que es autorizado por la esencia misma de una norma jurídica. Es decir, no se trata del análisis textual y plano del derecho, sino más bien lo que se busca es encontrar el contenido teleológico de la disposición. Estamos frente a un escenario donde lo que nos interesa es ir más allá del simple tenor literal de la norma, permitiéndole al operador jurídico, pero sobre todo al juez internacional, ejercer una facultad de aplicar una valoración jurídica a un caso concreto, lo que en otros términos podría ser una suerte de labor creativa, al llenar de contenido una disposición general y abstracta, que en el momento de su aplicación no cumple con su objetivo de cara al valor supremo de la justicia. Así las cosas, está completamente excluida la derogación de la ley por parte de la equidad infra legem, pues se trata de una situación absolutamente diferente, ya que la norma jurídica será aplicada pero imprimiéndole un contenido equitativo.

Bajo la misma lógica encontramos la resolución del Instituto de Derecho Internacional de fecha 3 de septiembre de 1937, en virtud de la cual se afirma:

$1^{\circ}$ que l'équité est normalement inhérente à une saine application du droit, et que le juge international, aussi bien que le juge interne, est, de par sa tâche même, appelé à en tenir compte dans la mesure compatible avec le respect du droit;

$2^{\circ}$ que le juge international ne peut s'inspirer de l'équité pour rendre sa sentence, sans être lié par le droit en vigueur, que si toutes les parties donnent une autorisation claire et expresse à cette fin $^{16}$. 
Al tenor del texto transcrito, es claro que se nos presentan dos posibles usos diferentes de la equidad por el juez internacional. El primero de ellos, relativo a la equidad infra legem, endilgándole al juez una labor interpretativa de la norma, siempre bajo los límites que le marca el derecho. Por su parte, el segundo supuesto se refiere más al uso del ex aequo et bono, en la medida que se podrá apartar el juez del derecho en vigor, siempre y cuando las partes lo hayan autorizado, incluso pudiendo llegar a pensar que puede estar permitiendo una equidad contra legem.

No obstante, hay que decir que las opiniones manifestadas por el Instituto de Derecho Internacional no son de especial aplicación para el tema de la delimitación marítima entre Estados, sino que más bien se trata un precepto de aplicación general en el derecho internacional. Así las cosas, parece más conveniente ir a la normas internacionales especializadas en la materia, para desentrañar con precisión el alcance de la equidad en el derecho internacional de delimitación marítima.

En este punto, queremos postular la siguiente afirmación: en el derecho internacional de la delimitación marítima, la equidad infra legem goza de una especial característica, cual es que esta no es el resultado del ejercicio interpretativo del juez internacional, sino que es la esencia misma de la norma, lo que implica que no son necesarios los esfuerzos de interpretación para fundamentar el uso de la equidad, pues esta es exigida directamente por el derecho convencional y consuetudinario aplicable, al prescribir que el fin de un procedimiento de delimitación marítima es obtener una equidad en los resultados.

Ahora bien, el argumento aquí presentado de ninguna manera busca darle especial cabida o servir de disfraz al uso del ex aequo et bono, ya que, como se ha mencionado en líneas anteriores, y a riesgo de ser reiterativo, esta posibilidad solamente es aceptada previa autorización de las partes, permitiendo así alejarse de manera absoluta de la norma internacional con vocación de aplicación. En ese entendido, se parte del cumplimiento a cabalidad del derecho, pues la norma es la equidad misma.

Para precisar aún más el papel de la equidad en los procedimientos de delimitación marítima es indispensable revisar lo que las normas convencionales han establecido, pero sobre todo el instrumento internacional de mayor importancia y acogida por la comunidad internacional actualmente en la materia ${ }^{17}$. La Convención de las Naciones Unidas sobre el Derecho del Mar

sion de Luxembourg, le 3 septembre 1937. Disponible en: http://www.idi-iil.org/idiF/resolutionsF/1937_lux_02_fr.pdf

17 Cabe resaltar que no es la única norma convencional existente sobre la materia, pero el día de hoy tratados internacionales como la Convención de Ginebra sobre Plataforma Continental de 1958 no son la fuente principal de controversias internacionales u objeto de discusiones doctrinales. 
de 1982, también conocida como CONVEMAR, UNCLOS (por sus siglas en inglés) o Convención de Montego Bay, comparte el interés de incluir la equidad en las reglas del derecho de delimitación marítima, estableciendo criterios de equidad para resolver los inconvenientes de superposición de derechos de los Estados con costas adyacentes o situadas frente a frente, reglamentación que se refiere al mar territorial ${ }^{18}$, a la zona económica exclusiva ${ }^{19}$ y a la plataforma continental ${ }^{20}$. Así las cosas, es claro que la equidad hace parte del espíritu de la Convención, lo que a su vez permite entender que el uso de este concepto no sólo está permitido, sino que es necesaria su utilización para cumplir con las expectativas de los Estados, cual es la solución equitativa.

Ahora, como lo mencionamos anteriormente, el problema de las normas convencionales es su efecto relativo, el cual se comprende de manera absoluta a través de dos principios generales del derecho internacional: pacta sunt

18 "Artículo 15. Delimitación del mar territorial entre Estados con costas adyacentes o situadas frente a frente. Cuando las costas de dos Estados sean adyacentes o se hallen situadas frente a frente, ninguno de dichos Estados tendrá derecho, salvo acuerdo en contrario, a extender su mar territorial más allá de una línea media cuyos puntos sean equidistantes de los puntos más próximos de las líneas de base a partir de las cuales se mida la anchura del mar territorial de cada uno de esos Estados. No obstante, esta disposición no será aplicable cuando, por la existencia de derechos históricos o por otras circunstancias especiales, sea necesario delimitar el mar territorial de ambos Estados en otra forma".

19 "Artículo 74. Delimitación de la zona económica exclusiva entre Estados con costas adyacentes o situadas frente a frente

"1. La delimitación de la zona económica exclusiva entre Estados con costas adyacentes o situadas frente a frente se efectuará por acuerdo entre ellos sobre la base del derecho internacional, a que se hace referencia en el artículo 38 del Estatuto de la Corte Internacional de Justicia, a fin de llegar a una solución equitativa.

"2. Si no se llegare a un acuerdo dentro de un plazo razonable, los Estados interesados recurrirán a los procedimientos previstos en la Parte XV.

"3. En tanto que no se haya llegado a un acuerdo conforme a lo previsto en el párrafo 1, los Estados interesados, con espíritu de comprensión y cooperación, harán todo lo posible por concertar arreglos provisionales de carácter práctico y, durante ese período de transición, no harán nada que pueda poner en peligro u obstaculizar la conclusión del acuerdo definitivo. Tales arreglos no prejuzgarán la delimitación definitiva.

"4. Cuando exista un acuerdo en vigor entre los Estados interesados, las cuestiones relativas a la delimitación de la zona económica exclusiva se resolverán de conformidad con las disposiciones de ese acuerdo".

20 "Artículo 83. Delimitación de la plataforma continental entre Estados con costas adyacentes o situadas frente a frente

"1. La delimitación de la plataforma continental entre Estados con costas adyacentes o situadas frente a frente se efectuará por acuerdo entre ellos sobre la base del derecho internacional, a que se hace referencia en el artículo 38 del Estatuto de la Corte Internacional de Justicia, a fin de llegar a una solución equitativa.

"2. Si no se llegare a un acuerdo dentro de un plazo razonable, los Estados interesados recurrirán a los procedimientos previstos en la Parte XV.

"3. En tanto que no se haya llegado al acuerdo previsto en el párrafo 1, los Estados interesados, con espíritu de comprensión y cooperación, harán todo lo posible por concertar arreglos provisionales". 
servanda y res inter alios acta. Así las cosas, la pregunta que surge es: ¿qué derecho debe aplicar el juez internacional frente a controversias de delimitación marítima, cuando a las partes o a alguna de ellas no se le hacen extensibles las obligaciones internacionales derivadas de las normas convencionales?

Pues bien, la Corte Internacional de Justicia se vio enfrentada a esta ausencia de norma convencional aplicable, en el caso de la plataforma continental del Mar del Norte, en una controversia que enfrentaba a la República Federal de Alemania, los Países Bajos y Dinamarca. Frente a la imposibilidad de aplicar la Convención de Ginebra de 1958 ya que Alemania no hacía parte del tratado, los Países Bajos y Dinamarca solicitaban la aplicación de la equidistancia en calidad de costumbre internacional, argumento que fue rechazado por la Corte, al afirmar que el uso obligatorio de la línea de equidistancia no hacía parte del derecho internacional consuetudinario. Y es debido a esta especial situación que la Corte, bajo el velo del derecho consuetudinario, construyó su propia norma bajo la fórmula "resultado equitativo-principios equitativoscircunstancias pertinentes". Es decir, la Corte, en vez de utilizar la norma del artículo 6.2 de la Convención de 1958 (pudiéndola haber declarado consuetudinaria), traducida en la fórmula "equidistancia-circunstancias especiales", va más allá, imprimiéndole un contenido teleológico a la delimitación marítima, cual es la equidad de resultados.

Pero esto no debe mirarse como un simple cambio de normatividad aplicable, puesto que el objetivo de obtener un resultado equitativo supone diferentes modificaciones, empezando porque la equidistancia no será ya el único método para la delimitación, teniendo entonces que observar diferentes aspectos derivados del caso en concreto, para determinar la forma cómo se llevará a cabo la asignación de dominios marinos a cada uno de los Estados. La regla de ese momento en adelante, como lo afirma Ruilboa García, ya no es la búsqueda de un único método de delimitación, sino un fin único: resultados equitativos ${ }^{21}$.

Ahora bien, la inquietud que surgió al momento del fallo comentado y que se ha mantenido en el tiempo se refiere a esa potestad o facultad creadora de normas en cabeza de la Corte Internacional de Justicia. Ciertamente los jueces tienen delimitadas sus funciones a la aplicación e interpretación del derecho, lo que a todas luces choca fuertemente con la posibilidad de crear derecho. No obstante, en el caso de la Corte Internacional de Justicia esta atribución parece derivar del artículo 38 de su Estatuto. Como lo mencionan Lucchini y Vœlckel, si bien la jurisprudencia es considerada como un medio auxiliar para la determinación de las normas de derecho, en ese ámbito cabe la posibilidad, no latente, pero al fin existente, de la creación de normas jurídicas ${ }^{22}$.

21 Ruiloba García, E. Circunstancias especiales y equidad en la delimitación de los espacios marítimos. En Real Instituto de Estudios Europeos. Zaragoza, 2001, 64.

22 Lucchini, L. y Veelckel, M. Droit de la mer. Paris: Pedone, 1990, 200. 
Esta creación de la Corte Internacional de Justicia tendría un uso prolongado en el tiempo, de tal manera que, una vez aparecida la Convención de Naciones Unidas sobre el derecho del $\operatorname{mar}^{23}$, sería la invención jurisprudencial la utilizada en los procesos de delimitación marítima y no la norma convencional, en razón a sus imprecisiones y vacíos. De acuerdo con Prosper Weil, los defectos recién mencionados, tienen como consecuencia que las normas de 1982 sean consideradas como simples directivas vagas y generales, de muy difícil aplicación a casos concretos ${ }^{24}$.

De esta manera podemos destacar una lista de casos en sede de la Corte Internacional de Justicia en que se aplica la fórmula "resultado equitativoprincipios equitativos-circunstancias pertinentes", tanto antes como después de la entrada en vigencia de la Convención de 1982. Así, podemos resaltar, entre otros, los diferendos de la plataforma continental entre Túnez y Libia (1982), la delimitación de la frontera marítima en la región del Golfo de Maine (1984), la plataforma continental entre Libia y Malta (1985), el diferendo fronterizo terrestre, insular y marítimo entre El Salvador y Honduras (1992), la delimitación marítima en la región situada entre Groenlandia y Jan Mayen entre Dinamarca y Noruega (1993), la delimitación marítima y cuestiones territoriales entre Qatar y Baréin (2001), la frontera terrestre y marítima entre Camerún y Nigeria (2002), el diferendo territorial y marítimo en el Mar Caribe entre Nicaragua y Honduras (2007), la delimitación marítima en el Mar Negro (2009), el diferendo territorial y marítimo entre Nicaragua y Colombia (2012) y el diferendo marítimo entre Perú y Chile (2014).

De acuerdo con todo lo anterior, consideramos necesario acudir a la jurisprudencia internacional y conocer la metodología que la Corte Internacional de Justicia ha venido aplicando a los diferentes diferendos de delimitación marítima.

\section{LA OPERACIÓN ADJUDICATIVA DE DELIMITACIÓN CON EQUIDAD}

La Corte Internacional de Justicia a lo largo de su jurisprudencia ha ido construyendo, mediante el uso de la equidad infra legem, todo un procedimiento para llevar a cabo la delimitación de los espacios marítimos de Estados que se encuentran en controversia. Por supuesto, pese a que existe una uniformidad en la manera de fallar del Tribunal, creando una suerte de jurisprudencia constante, todos y cada uno de los casos resultan únicos, con especificidades especiales que deben ser tenidas en cuenta en el momento de fallar, lo que no impide entonces que se tracen directrices generales que serán analizadas y

23 La Convención fue aprobada el 30 de abril de 1982 pero su entrada en vigor solamente se verificó el 16 de noviembre de 1994, un año después de la 60. a ratificación.

24 WeIL, P. Perspectives du droit de la délimitation maritime. Paris: Pedone, 1988, 12. 
escogidas por la Corte al realizar la operación adjudicativa de delimitación, siempre bajo la idea de la equidad de resultados.

Ahora bien, como lo mencionamos en el aparte anterior, es indispensable, para el cumplimiento de la labor encomendada a la Corte, la identificación del derecho aplicable, teniendo presente que es posible que entre las partes de la controversia limítrofe existan tratados internacionales de delimitación marítima, o escenarios que permitan desentrañar la existencia de una aquiescencia o estoppel ${ }^{25}$, o incluso, de una costumbre entre los Estados especialmente concernidos. En estos casos se trata de normas convencionales o consuetudinarias particulares, situación diferente a la que se puede observar cuando se es parte, por ejemplo, de la Convención de Montego Bay, puesto que los Estados que alegan la existencia de acuerdos previos buscarán demostrar que no hay lugar a construir una hipótesis de delimitación, en tanto la situación ya ha sido convencionalmente solucionada o de la actuación de las partes se muestra la existencia de acuerdos "tácitos" sobre el particular ${ }^{26}$.

Una vez analizadas las situaciones mencionadas, si la Corte encuentra que no hay mérito para conservar las alegaciones de los Estados sobre la existencia de acuerdos escritos o prácticas estatales que den noticia de una delimitación entre las partes, procede a determinar el derecho aplicable en las normas jurisprudenciales que se han ido construyendo, bajo la trilogía de línea media provisional, circunstancias pertinentes y análisis de desproporcionalidad, todo en el marco de la equidad como principio teleológico, en busca de un resultado equitativo.

\subsection{Linea media provisional}

Antes de iniciar el procedimiento de delimitación, la Corte tiene una tarea preliminar que evacuar, y es la identificación de las costas relevantes ${ }^{27}$, entendidas como aquellas que le pertenecen a un Estado, las cuales tienen la virtualidad de generar proyecciones que se superponen con las proyecciones

25 International Court of Justice. Case concerning the Temple of Preah Vihear, Merits, Judgements, ICJ Reports 1962, 23.

26 International Court of Justice. Différend maritime (Pérou/Chili), Judgement, 2014, pr. 24-151; International Court of Justice. Territorial and maritime dispute (Nicaragua/Colombia), Judgment, ICJ Reports 2012, pr. 40-65; International Court of Justice. Case concerning territorial and maritime dispute between Nicaragua and Honduras in the Caribean sea, Judgemt, ICJ Reports 2007, p. 69. International Court of Justice. Différend maritime (Pérou/Chili), Judgement, 2014, 65 .

27 "First it is necessary to identify the relevant coast in order to determine what constitutes in the specific context of a case the overlapping claims to these zones. Second, the relevant coasts need to be ascertained in order to check, in the third and final stage of teh delimitation procces, whether any disproportionality exists in the ratios of the coastal legth of each State and the maritime areas falling either side of the delimitation line": International Court of Justice. Case concerning maritime delimitation in the Black Sea, Judgment, ICJ Reports 2009, 27. 
de otro Estado. Tal situación solamente se entiende cuando se parte de la idea de que el título que tiene un Estado costero sobre los espacios marinos se cimienta en el principio de adyacencia, es decir, la tierra domina al mar, y por tal razón, un Estado que posea costas tiene el derecho de reclamar soberanía sobre las aguas del mar que lo bordean. Bajo ese entendido, es posible que en el momento en que los Estados reivindiquen soberanía sobre alguno de los espacios marinos sus derechos entren en controversia con los derechos de otro Estado.

Así las cosas, se tendrán como costas relevantes aquellas que generen proyecciones que se superpongan con las proyecciones de otro Estado, lo que a su vez significa que aquellas que no se superpongan por ninguna razón, deberán no ser tenidas en cuenta ${ }^{28}$. Ahora bien, determinadas las costas relevantes, la Corte deberá establecer cuáles son los puntos de base a partir de los cuales se trazará una línea media provisional, advirtiendo que es posible que se excluyan algunos accidentes geográficos para la selección de los puntos relevantes, ya sea por su pequeño tamaño o por la distancia a la que se encuentran, todo esto bajo la idea de evitar una remodelación geográfica judicial no autorizada ni por el derecho ni por la práctica internacional, y además, para no ir en contra de la obtención de un resultado equitativo del procedimiento ${ }^{29}$.

Una vez determinados los puntos de base se deberá proceder a la utilización de un método objetivo de delimitación, constituyendo la regla general, mas no obligatoria $^{30}$, la equidistancia. Esta supone la división en partes iguales de las áreas de superposición, llegando normalmente a un resultado equitativo. Pero como lo acabamos de mencionar, no es la única forma de llegar a una línea media provisional, ya que la regla general de la equidistancia encuentra dos excepciones que las partes deben solicitar y probar la necesidad de utilizar alguno de estos métodos, cuales son la bisectriz y los segmentos de ajuste.

Para circunstancias especiales, como es el caso de las costas adyacentes, es posible y además necesario que se utilice un mecanismo subsidiario a la equidistancia, como es el método de la bisectriz. Así lo podemos observar en el caso de la disputa marítima y territorial entre Nicaragua y Honduras, de cara a la presencia de una concavidad de la costa, situación que impedía fijar los puntos de base en el término del límite terrestre en el mar, situado en el delta del río Coco, donde por la existencia de acreción de sedimentos

28 International Court of Justice. Continental shelf (Tunisia/Libya), ICJ Reports 1982, pr. 61,75 .

29 International Court of Justice. Case concerning maritime delimitation in the Black Sea, Judgment, ICJ Reports 2009, 110; International Court of Justice. Territorial and maritime dispute (Nicaragua/Colombia), Judgment, ICJ Reports 2012, 78-79.

30 "Sufficeit to say that a rule may be a rule, even a paramount rule, and yet not have to be 'compulsory applied in all situations'". International Court of Justice. Continental shelf (Tunisia/Libya), ICJ Reports 1982, 194, pr. 60. 
se presentó un desplazamiento de la desembocadura del río y por ende del punto de término. Tratándose de la bisectriz, el uso de la equidad puede estar mucho más influenciado por elementos subjetivos, ya que es necesario determinar la dirección general de la costa, así como precisar cuál sección de la costa será tenida en cuenta para reflejar la dirección y, finalmente, decidir la escala del mapa que se utilizará, aspecto que podría causar en la apreciación de la dirección de la costa una modificación.

Por otra parte, se han utilizado otros métodos subsidiarios llamados soluciones intermedias, para referirse a los segmentos de ajuste, en virtud de los cuales ciertos sectores del límite se ajustan de manera tal que se puede encajar un límite previo con la equidistante, tal como se puede observar en la controversia fronteriza entre Camerún y Nigeria ${ }^{31}$.

Por último, y no menos importante, es indispensable que la construcción de esta línea media provisional se haga de acuerdo con los principios equitativos, máximas que también han sido desarrolladas por la jurisprudencia de la Corte Internacional de Justicia. Sin el ánimo de restarles importancia a los mismos, los mencionaremos absteniéndonos de entrar en detalle en su contenido, pues la simple enunciación deja claridad sobre su significado. Así entonces, tenemos:

- Principio de la no penetración en la prolongación natural de otro Estado ${ }^{32}$;

- Principio de la división por igual de las áreas de superposición ${ }^{33}$;

- Principio de la inexistencia de un método de delimitación obligatorio ${ }^{34}$;

- Principio de la no remodelación total de la geografía ${ }^{35}$;

- Principio de la no equivalencia necesaria entre equidad e igualdad/ proporcionalidad $^{36}$.

Finalizada la construcción de la línea media provisional de acuerdo a las especificidades del caso concreto, será necesario pasar al segundo punto del procedimiento de delimitación.

31 International Court of Justice. Land and maritime boundary between Cameroon and Nigeria (Equatorial Ginea intervening), Judgment, ICJ Reports 2002, 441, pr. 307.

32 International Court of Justice. North sea continental shelf, Judgment, ICJ Reports $1969,53$.

33 Ibíd.; International Court Of Justice. Delimitation of the maritime boundary in the Gulf of Maine Area, Judgment, ICJ Reports 1984, 327.

34 International Court of Justice. Continental shelf (Tunisia/Libya), ICJ Reports 1982, 47, pr. 63.

35 International Court of Justice. North sea continental shelf, Judgment, ICJ Reports 1969, 49; International Court Of Justice. Case concerning maritime delimitation in the Black Sea, Judgment, ICJ Reports 2009, 45, pr. 149; International Court of Justice. Case concerning territorial and maritime dispute between Nicaragua and Honduras in the Caribean sea, Judgemt, ICJ Reports 2007, 79, pr. 289.

36 International Court of Justice. Différend maritime (Pérou/Chili), Judgement, 2014, 65, pr. 193. 


\subsection{Circunstancias pertinentes}

Una vez establecida la línea media provisional, la Corte debe examinar si debido a la existencia de factores objetivos se debe ajustar o desplazar la misma, con el fin de llegar a un resultado equitativo, aunque puede ocurrir que no sea necesario hacer modificaciones ${ }^{37}$. Estos factores, conocidos como circunstancias pertinentes, tienen la función de permitir que la línea media provisional trazada por alguno de los métodos habilitados para esta tarea no sea inequitativa, es decir, que por circunstancias particulares el resultado al que se haya llegado no cumpla con el objetivo de la delimitación marítima ${ }^{38}$.

De cierta manera el uso de las circunstancias pertinentes lo podemos traducir en un uso de la equidad correctiva, ya que los resultados obtenidos a través de la equidistancia o los métodos subsidiarios no han permitido llegar a un resultado equitativo, de tal manera que tener en cuenta estos aspectos busca modificar los resultados preliminares materializando la equidad infra legem.

La Corte Internacional de Justicia ha definido las circunstancias pertinentes como hechos o realidades que necesariamente deben tenerse en cuenta en el proceso de delimitación, en la medida que incidan en la obtención de un resultado equitativo ${ }^{39}$. Estas comprenden todo un catálogo no taxativo de situaciones que comprenden los factores geográficos y geomorfológicos, los factores socioeconómicos, circunstancias relacionadas con la seguridad e incluso la conducta misma de las partes. Debido a la diversa naturaleza que poseen las circunstancias pertinentes, podríamos clasificarlas en geográficas y no geográficas, considerando que las primeras parecen resultar de mayor envergadura, en el momento de la modificación de la línea provisional, que aquellas que no obedecen realmente a criterios territoriales. Aunque la última afirmación se trata de una percepción personal, es posible corroborar de manera objetiva la situación, haciendo un barrido jurisprudencial en materia de delimitación marítima ante la Corte.

Dentro de las circunstancias pertinentes más usadas en los fallos de la Corte, así como en las alegaciones de los Estados en controversia, podemos destacar a las siguientes, aclarando que no se trata de una lista taxativa, ni mucho menos exhaustiva.

Configuración de las costas ${ }^{40}$. Su importancia es latente y es la consecuencia lógica del título que detentan los Estados sobre el mar, el llamado

37 International Court of Justice. Différend maritime (Pérou/Chili), Judgement, 2014, 65, pr. 191-192.

38 International Court of Justice. Case concerning maritime delimitation in the Black Sea, Judgment, ICJ Reports 2009, 112, pr. 155.

39 International Court of Justice. Land and maritime boundary between Cameroon and Nigeria (Equatorial Ginea intervening), Judgment, ICJ Reports 2002, 62, pr. 55.

40 Sobre el particular destacamos los siguientes casos: Délimitation maritime dans la región située entre le Groenland et Jan Mayen; Delimitation of the maritime boundary in the 
principio de adyacencia. Podríamos afirmar que esta circunstancia se debe analizar siempre bajo el principio de la no remodelación total de la geografía, y será analizada sobre todo cuando existen configuraciones costeras cóncavas o convexas, independientemente del método de delimitación que se haya escogido.

Longitud de la costa y proporcionalidad de la misma ${ }^{41}$. En ciertos eventos se puede encontrar una gran desproporción entre las costas relevantes de los Estados en controversia, situación que por supuesto no puede ser analizada bajo el principio de división por igual de las áreas de superposición, so pena de incurrir en un resultado flagrantemente inequitativo. Ejemplo claro de esta situación de desproporción y por ende de la necesidad de usar la equidad correctiva lo encontramos en el fallo de la disputa territorial y marítima entre Nicaragua y Colombia, donde la longitud de las costas pertinentes es de 531 $\mathrm{km}$ para Nicaragua y de $65 \mathrm{~km}$ para Colombia ${ }^{42}$, razón por la cual era latente la modificación de la línea media provisional.

Presencia de islas, rocas y elevaciones de bajamar ${ }^{43}$. Todas las situaciones en mención presentan inconvenientes de no muy fácil solución, pero sin lugar a dudas la circunstancia de mayor dificultad es la presencia de islas, como por ejemplo cuando estas se encuentran realmente alejadas del territorio principal del Estado, como los casos de Jan Mayen y St. Pierre-et-Miquelon. Igualmente resulta de difícil solución cuando las islas se encuentran ubicadas en el lado equivocado del límite marítimo entre dos territorios principales, llevando a situaciones de enclaves. Asimismo revisten gran dificultad las elevaciones de bajamar, como ocurrió en el caso de la delimitación entre Qatar y Baréin, o bien las islas que pueden llegar a ser consideradas como rocas. Sea cual sea la situación particular, es importante que estas presencias no causen una inequidad en los resultados, ya sea porque se tuvieron en consideración o porque se ignoraron, causando en cualquier escenario un perjuicio para alguna de las partes.

La geología y la geomorfología ${ }^{44}$. Aunque la conformación natural de los territorios resultaría una circunstancia de especial observancia, hay que decir que hoy día la jurisprudencia ha dejado a un lado los factores geológicos y

Gulf of Maine Area; North Sea continental shelf; Continental shelf (Tunisia/Libya); Land and maritime boundary between Cameroon and Nigeria; Case concerning maritime delimitation in the Black Sea.

41 Sobre el particular destacamos los siguientes casos: Continental shelf (Libya/Malta); Continental shelf (Tunisia/Libya); Territorial and maritime dispute (Nicaragua/Colombia).

42 International Court of Justice. Territorial and maritime dispute (Nicaragua/Colombia), Judgment, ICJ Reports 2012, pr. 153.

43 Sobre el particular destacamos los siguientes casos: Continental shelf (Libya/Malta); Case concerning maritime delimitation in the Black Sea; North Sea continental shelf; Territorial and maritime dispute (Nicaragua/Colombia); Continental shelf (Tunisia/Libya); Continental shelf (Libya/Malta).

44 Sobre el particular destacamos los siguientes casos: North Sea continental shelf; 
geomorfológicos en casos sobre la delimitación de la plataforma continental, reemplazando dichos aspectos por el criterio de la distancia en detrimento de la batimetría y la explotabilidad. No quiere decir esto que se haya convertido en una regla general de obligatorio cumplimiento, sino más bien parecería una tendencia, pues algunos fallos nos han mostrado cómo continúan vigentes los aspectos geológicos y geomorfológicos ${ }^{45}$. Es así como, bajo los criterios de batimetría y explotabilidad, se parte de que el elemento fáctico de mayor relevancia para llevar a cabo la delimitación es que la plataforma continental sea considerada como la prolongación natural de dos o más Estados, argumento que permite entrar en la discusión de la plataforma continental extendida.

Por otra parte encontramos aquellas circunstancias relevantes que no obedecen a un criterio geográfico, pero que igualmente pueden ser tenidas en cuenta en el momento del procedimiento de delimitación marítima. Así las cosas, tenemos:

La economía y los recursos naturales ${ }^{46}$. Esta circunstancia es de suma importancia cuando la delimitación se circunscribe a la zona económica exclusiva y/o a la plataforma continental, debido al contenido eminentemente económico que envuelve los elementos en mención. La explotación de los recursos naturales renovables y no renovables mueve fuertemente los intereses de los Estados, teniendo que observar en el momento de la delimitación la actividad de los Estados en controversia con respecto al uso y manejo de los recursos que en las zonas en disputa se encuentran.

La economía tradicional ${ }^{47}$. Junto con la anterior circunstancia, esta debe ser analizada a través de la práctica de los Estados en las zonas en disputa, teniendo en cuenta que algunas circunstancias obedecen a las actividades tradicionales que sustentan a las comunidades costeras. Esta situación que se materializa sobre todo en las pesquerías tradicionales no ha llegado a constituirse como una circunstancia relevante con virtualidad correctiva.

La posición socioeconómica de los Estados ${ }^{48}$. Algunas controversias de delimitación se suscitan entre Estados cuyas condiciones económicas son completamente diferentes, y una división de las zonas marítimas en disputa puede afectar gravemente la posición de alguna de las partes, ya que puede

Continental shelf (Tunisia/Libya); Case concerning territorial and maritime dispute between Nicaragua and Honduras in the Caribean sea.

45 International Court of Justice. Continental shelf (Tunisia/Libya), ICJ Reports 1982, 48-49.

46 Sobre el particular destacamos los siguientes casos: North Sea continental shelf; Continental shelf (Tunisia/Libya); Continental shelf (Libya/Malta); Delimitation of the maritime boundary in the Gulf of Maine Area; Délimitation maritime dans la región située entre le Groenland et Jan Mayen.

47 Sobre el particular destacamos el siguiente caso: Case concerning maritime delimitation and territorial questions between Qatar and Bahrain;

48 Sobre el particular destacamos el siguiente caso: Continental shelf (Tunisia/Libya); Délimitation maritime dans la región située entre le Groenland et Jan Mayen. 
resultar en la pérdida de dominios marinos que les proveen recursos naturales, y que a su vez aportan de manera importante en su balanza comercial. Aunque en la jurisprudencia se ha discutido el tema, está completamente claro que la circunstancia en mención no se ha aceptado como una de aquellas con contenido correctivo.

La presencia de Estados fuera de la controversia ${ }^{49}$. Como resulta obvio, una delimitación marítima entre dos o más Estados solo puede tener efecto en los territorios sobre los cuales estos tienen vocación de soberanía. Frente a la presencia de terceros Estados, la Corte Internacional de Justicia ha dejado claro que aquellos dominios se encuentran por fuera del alcance de su decisión, razón por la cual difícilmente puede esta circunstancia tener un efecto correctivo en el momento del ajuste a la línea media provisional de la delimitación.

La seguridad y defensa de los Estados ${ }^{50}$. No se puede olvidar que entre los fines del ordenamiento jurídico internacional se encuentran la protección de la independencia política y la integridad territorial de los Estados. Pues bien, este objetivo debe ser observado en cualquier momento y bajo cualquier circunstancia, razón por la cual la Corte Internacional de Justicia ha reconocido que las consideraciones de seguridad sí tienen la posibilidad de influir en una delimitación marítima de acuerdo con el criterio de proximidad, de tal manera que se puede modificar la línea media provisional para ajustar de manera equitativa la delimitación con la seguridad y defensa de los Estados.

Una vez analizadas y aplicadas las circunstancias pertinentes, de acuerdo con las alegaciones de los Estados en controversia, así como las que por las particularidades del caso concreto deban tenerse en cuenta, la Corte procederá, de ser necesario, a ajustar la línea media provisional, de tal manera que tengamos, de acuerdo con las prescripciones de la equidad infra legem, un resultado equitativo. Finalizado esto, la Corte pasará al último paso del procedimiento.

\subsection{El análisis de desproporcionalidad}

Construida la línea limítrofe definitiva, sin importar si se ajustó o no con alguna o varias de las circunstancias pertinentes, la Corte debe verificar si

49 Sobre el particular destacamos los siguientes casos: North Sea continental shelf; Continental shelf (Tunisia/Libya); Land, island and maritime frontier dispute (El Salvador/Honduras), Application to intervene; Land and maritime boundary between Cameroon and Nigeria; Case concerning territorial and maritime dispute between Nicaragua and Honduras in the Caribean sea.

50 Sobre el particular destacamos los siguientes casos: Continental shelf (Libya/Malta); Délimitation of the maritime boundary (Guinea/Guinea-Bissau); Délimitation maritime dans la región située entre le Groenland et Jan Mayen; Case concerning maritime delimitation in the Black Sea. 
el resultado de la delimitación es equitativo, labor que ha sido llamada a lo largo de su jurisprudencia como análisis o examen de desproporcionalidad, cuyo objeto es determinar la existencia de elementos que no permitan una delimitación adecuada en términos de proporcionalidad, cuyo efecto genera un resultado inequitativo. Por supuesto el objeto del análisis se concentrará en las áreas asignadas a cada una de las partes en conflicto, dentro de la zona de superposición de sus derechos. No tendrá la Corte por qué analizar otras circunstancias que no sean parte del gel contencioso del caso concreto.

Aunque parece una tarea sencilla debido a que debe ser orientada por los principios equitativos, es una labor que presenta dificultades; sobre todo resulta ampliamente criticada por los Estados en la medida que sus pretensiones, en algunos casos exageradas, no son avaladas por los resultados, todo esto debido a que en ocasiones se tiende a confundir equidad con igualdad.

Para este análisis final, la Corte deberá tener en cuenta todos y cada uno de los aspectos que determinaron el resultado final, desde la determinación de las costas relevantes, así como su longitud, hasta llegar a las circunstancias pertinentes.

Una vez surtida esta etapa, la Corte deberá ajustar el resultado en caso de encontrar una desproporción marcada ${ }^{51}$. De igual manera es preciso entender que este análisis y ajuste no supone una aplicación estricta del principio de proporcionalidad, en la medida que difícilmente existirá una perfecta correlación entre la longitud de las costas pertinentes y la parte de la zona que le fue atribuida a cada uno de los Estados ${ }^{52}$.

Como podemos ver, todo el procedimiento que realiza la Corte de cara a una delimitación marítima entre Estados con derechos que se superponen obedece definitivamente al criterio de equidad, pues en todas y cada una de las etapas el tribunal analiza en qué medida puede la decisión tornarse inequitativa y busca la forma de corregirla.

Asimismo resulta claro que desde el año 1969 la Corte Internacional de Justicia ha utilizado la fórmula "línea media provisional, circunstancias pertinentes y análisis de desproporcionalidad", adaptándola a las particularidades de cada caso, lo que ha permitido esclarecer algunos puntos y enriquecer la teoría de la delimitación marítima a nivel judicial.

Finalmente, es completamente claro que los fallos proferidos por la Corte Internacional de Justicia en la materia en examen reciben amplias críticas, pero también son bien recibidos por algunos. Creemos que lo anterior se debe en principio a los intereses que tenga cada observador de la decisión de la Corte, pero en términos generales consideramos que se trata de una posición

51 International Court of Justice. Territorial and maritime dispute (Nicaragua/Colombia), Judgment, ICJ Reports 2012, pr. 240.

52 International Court of Justice. Continental shelf (Libya/Malta), ICJ Reports 1985, 45, pr. 58. 
jurisprudencial ampliamente aceptada, en la medida que un buen número de casos se ha resuelto bajo este tipo de delimitación judicial sin generar mayores inconvenientes en los miembros de la comunidad internacional.

\section{CONCLUSIONES}

El derecho internacional de la delimitación marítima ha evolucionado con el paso del tiempo, debido a la incesante voluntad de los Estados de extender su soberanía en el territorio marítimo y así poder disfrutar de un mayor poder soberano, pero sobre todo de los recursos naturales que en los dominios marinos se encuentran. Por esta razón, y con el fin de evitar que los conflictos entre Estados proliferen, la comunidad internacional ha tratado de establecer reglas convencionales que permitan delimitar los espacios marinos, lo que, pese a estar respaldado por una buena intención, parece no haber alcanzado un mayor éxito, causando entonces importantes controversias en el derecho internacional.

Ante la situación mencionada, el derecho consuetudinario, pero más que este, la labor del juez internacional y, en especial, la jurisprudencia de la Corte Internacional de Justicia, ha venido a paliar los efectos causados por la ausencia de claridad y efectividad de la regulación internacional de la delimitación marítima, creando una fórmula basada en la equidad infra legem, según la cual todo el procedimiento se debe realizar bajo la idea de obtener una equidad de resultados. La trilogía propuesta por la Corte, es decir, "línea media provisional, circunstancias pertinentes y análisis de desproporcionalidad", corresponde a etapas que no deben analizarse de manera individual e independiente, pues todas ellas comparten un thelos, aspecto primordial de la construcción jurisprudencial y que simplemente se reduce a un resultado equitativo.

Ahora bien, analizados estos puntos se puede considerar que varias de las críticas que se le han formulado a la metodología jurisprudencial de la delimitación marítima se fundan en una desconfianza hacia el término equidad, relacionándolo indefectiblemente con el concepto de ex aequo et bono, figura también aceptada bajo ciertas condiciones por el derecho internacional pero cuyo principal efecto o defecto (según la posición que se asuma) será la exclusión absoluta de la aplicación de las normas del derecho, dejando pues la solución de controversias al buen sentido de justicia del juez internacional.

Entendemos por supuesto la preocupación, que además en América Latina se ha hecho sentir en los últimos años, con ocasión de los tres fallos de delimitación emitidos por la Corte Internacional de Justicia entre países de la región, la cual se ha fundamentado en la afirmación de un excesivo subjetivismo, que afecta enormemente los intereses de algunos Estados. Sin embargo, no compartimos aquellas posiciones que satanizan el uso de la equidad en los procesos de delimitación, pues, como se puede observar 
en el desarrollo de este escrito, si bien hay decisiones subjetivas del juez en algunos momentos, el contenido objetivo del procedimiento es mayoritario.

Como queda claro en este texto, la equidad infra legem no posee las cualidades de la contra legem, de hecho son completamente contradictorias, razón por la cual aseverar que la equidad utilizada en los procedimientos de delimitación marítima excluye la norma jurídica es un error. De nuevo y además como conclusión del presente trabajo, la equidad aquí estudiada es aquella que yace del espíritu mismo de la disposición jurídica, no siendo una invención del juez internacional a través de la costumbre, pues como se mencionó, también es el objetivo de las normas convencionales. Así las cosas, el camino nos lleva inevitablemente a una afirmación, y es que los Estados como sujetos del derecho internacional y, por ende, como participantes activos en la creación de normas convencionales y consuetudinarias, quieren que la equidad esté presente en sus procesos de delimitación marítima, pues de no ser así, no se ratificarían tratados como la CONVEMAR y/o se denunciarían estos, pero además, en la práctica, los Estados cambiarían su modus operandi en las relaciones internacionales de tipo marítimo.

Finalmente, no podemos olvidar que la relación existente entre derecho y justicia encuentra un gran aliado en la equidad, de tal manera que esta le permite a un instrumento creado por el hombre para regular sus relaciones sociales acercarse en mayor medida al valor supremo de la justicia, formulación que por supuesto se puede hacer extensiva al derecho internacional sin alterar en nada su esencia. Así pues, y como última gran conclusión, la equidad correctiva, frecuentemente aquí mencionada, no busca nada diferente en el derecho internacional de la delimitación marítima que proveer decisiones basadas en la justicia.

\section{REFERENCIAS}

Anderson, D. (2008). Modern Law of the Sea: Selected Essays. Leiden: Martinus Nijhoff.

Aristóteles. (2011). Ética a Nicómaco. Madrid, Tecnos.

Bardonnet, D., dir. (1983). Le nouveau droit de la mer. Paris: Pedone.

Cour International de Justice. Délimitation maritime dans la región située entre le Groenland et Jan Mayen (Danemark/Norvège), Arrêt, 14 juin 1993, Recueil CIJ 1993, opinión individuelle M. Weeramantry, § 51 .

Dupuy, R.-J. (1985). Traité du nouveau droit de la mer. Paris: Economica-Bruylant.

Falcón y Tella, M. J. (2005). Equidad, derecho y justicia. Madrid: Centro de estudios Ramón Areces.

Franck, T. (1995). Fairness in International Law Institutions. Oxford: Oxford University Press. 
Grocio, H. (1979). De la libertad de los mares. Trad. V. Blanco García y L. García Arias. Madrid: Centro de Estudios Constitucionales.

Institute du Droit International. La compétence du juge international en équité. Session de Luxembourg, le 3 septembre 1937. Disponible en: http://www.idi-iil.org/idiF/ resolutionsF/1937_lux_02_fr.pdf

International Court of Justice. (2009). Case concerning maritime delimitation in the Black Sea, Judgment, ICJ Reports.

International Court of Justice. (2007). Case concerning territorial and maritime dispute between Nicaragua and Honduras in the Caribean sea, Judgemt, ICJ Reports, 79, pr. 289.

International Court of Justice. (1962). Case concerning the Temple of Preah Vihear, Merits, Judgements, ICJ Reports.

International Court of Justice. (1985). Continental shelf (Libya/Malta), ICJ Reports.

International Court of Justice. (1982). Continental shelf (Tunisia/Libya), Judgement, ICJ Reports.

International Court of Justice. (1984). Delimitation of the maritime boundary in the Gulf of Maine Area, Judgment, ICJ Reports.

International Court of Justice. (2014). Différend maritime (Pérou/Chili), Judgement.

International Court of Justice. (2002). Land and maritime boundary between Cameroon and Nigeria (Equatorial Ginea intervening), Judgment, ICJ Reports.

International Court of Justice. (1969). North sea continental shelf, Judgment, ICJ Reports.

International Court of Justice. (2012). Territorial and maritime dispute (Nicaragua/ Colombia), Judgment, ICJ Reports.

Lucchini, L. y Velckel, M. (1990). Droit de la mer. Paris: P edone.

OrRego Vicuña, F. (1991). El papel de la Corte Internacional de Justicia y otros tribunales en el desarrollo de la delimitación marítima. Estudios Internacionales. Vol. 24, n. ${ }^{\circ} 95$.

Pirote, O. (1973). La notion d'équité dans la jurisprudence récente de la cour international de justice. RGDIP. Paris: t. 77, /1.

Rawls, John. (1997). Teoría de la justicia. México: FCE.

Rodríguez Cuadros, M. (2007). Delimitación marítima con equidad: el caso de Perú y Chile. Lima: Peisa.

Ruiloba García, E. (2001) Circunstancias especiales y equidad en la delimitación de los espacios marítimos. En Real Instituto de Estudios Europeos, Zaragoza.

VÁsquez Carrizosa, A. (1986). El nuevo derecho del mar. Evolución y proyecciones económicas. Bogotá: Temis.

Weil, Prosper. Perspectives du droit de la délimitation maritime. Paris: Pedone, 1988. 